\title{
Correction to: Explanation as a Process: User-Centric Construction of Multi-level and Multi-modal Explanations
}

Bettina Finzel, David E. Tafler, Stephan Scheele, and Ute Schmid

Correction to:

Chapter "Explanation as a Process: User-Centric Construction of Multi-level and Multi-modal Explanations" in: S. Edelkamp et al. (Eds.): KI 2021: Advances in Artificial Intelligence, LNAI 12873, https://doi.org/10.1007/978-3-030-87626-5_7

The original version of this chapter was inadvertently published with a misspelling in the explanatory dialogue of Fig. 5. It has been updated as follows:

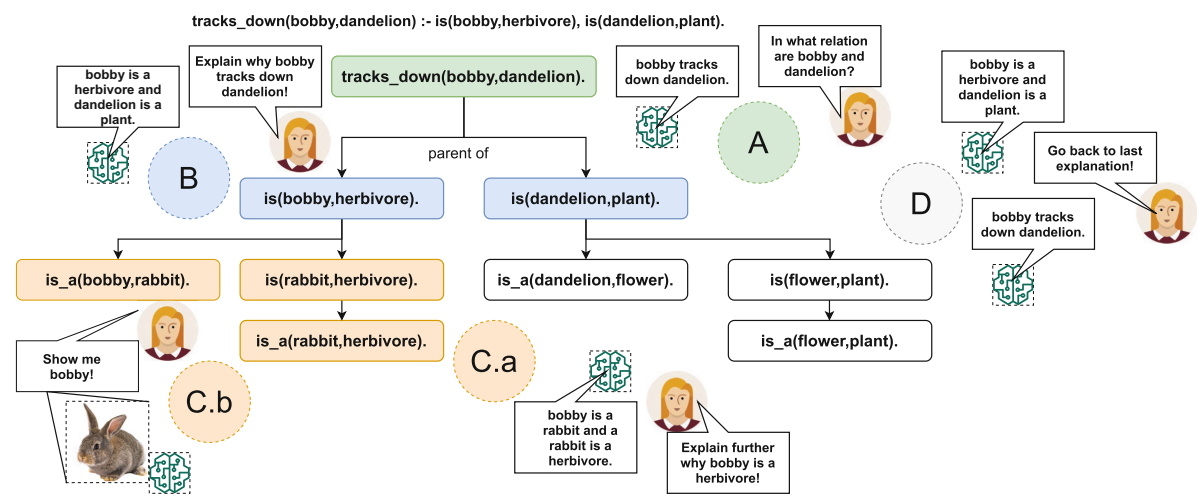

Fig. 5. An explanatory tree for tracks_down(bobby, dandelion), that can be queried by the user to get a local explanation why Bobby tracks down dandelion (steps A and B). A dialogue is realized by different drill-down questions, either to get more detailed verbal explanations or visual explanations (steps C.a and C.b)). Furthermore, the user can return to the last explanation (step D). 